\title{
Existence of Nano Level Force in Protein Plays Applications of Maximum Untold Understanding of Life Form
}

\author{
Rajasekaran Ekambaram, R. Meenal, Prawin Angel Michael, R. Indupriya
}

\begin{abstract}
There are many interactive forces between atoms applied to solve the problem of nature of molecules. One would go on applying this to several diseases and sufferings. On doing so, we have discovered that a new dimension of atomic arrangements playing a role in existence of force of interaction at nano level say at $1.6 \mathrm{~nm}$. Arrangements are in such way that it maintains a carbon fraction of 0.3144 in the structure of biologically important molecule called protein. Arrangements are important from maintaining structure and another way of interaction due to the deficiency of this domain formation. All our analysis conclude that there is new kind of force of attraction available for advancing the science here in biology and other field as well as other elements possess this nano level forces of attraction. Our results are validated with crystal availability because of force existence. Otherwise other may have to be studied accordingly. Bond of all atoms involved in domain formation are altered from original value of bond formation but increased or decreased according to the type of bonds. Alteration can be a measure of this newly identified nano level force of interaction. Our analysis can be extended to other problems in our science of untold answer.

Keywords : atomic force, carbon nano level network, crystal analysis, nano force.
\end{abstract}

\section{INTRODUCTION}

Maintaining the molecular structure intact is given priority to the van der Waals and electrostatic interaction. Overwhelmingly coherent nature of molecule existence has to be interpreted from forces arising from these non-bonded and bonded interactions. Available sources of interaction reveal that adequacy is not met with solving all of our problems associated to sufferings. Adequacy in the sense that all those of human value maintaining the carbon role adequately are not addressed. Our idea of conversion of arrangement of carbon profile may be of useful from nearing the untold answer to the human sufferings. Our idea was to go back a decade of carbon analysis finding maintenance of carbon value within the system of biologically important protein molecule which was analyzed after careful observation that protein attempting to maintain fraction of carbon 0.3144 at all level [1]. Arrangements are in such a way

Revised Manuscript Received on December 30, 2019.

* Correspondence Author

Rajasekaran Ekambaram, V.S.B. Engineering College, Karur-639111, Tamilnadu, India. Email: ersekaran@gmail.com

R. Meenal*, Prawin Angel Michael, Karunya Institute of Technology and Sciences, Coimbatore - 641114, Tamilnadu, India. Email: meenasekar5@gmail.com, prawin@karunya.edu

R.Indupriya, Kazan Federal University, Kazan-420012, Tatarstan, Russia. Email: indupriyarsekaran@gmail.com

(C) The Authors. Published by Blue Eyes Intelligence Engineering and Sciences Publication (BEIESP). This is an open access article under the CC BY-NC-ND license (http://creativecommons.org/licenses/by-nc-nd/4.0/) that aminoacid composition arranged according to the need based of carbon profile of law of rule. Otherwise call it as carbon profile of rule of law for maintenance and advancement progressively in nature of interaction [2]. Overall performance were monitored and analyzed according to the function and availability all along the sequence [3] and 3D structure [4] of several existing protein molecules. Infact other molecules were also subjected to this study of interaction. Analyses were thorough in all sense that arbitrary arrangements are according to the nature of interaction accordingly the protein is. Otherwise one concluded that proteins are essentially a follower of adequacy principle of carbon value. Accordingly alterations are carried out to meet out the role of proteins in the system of response. Overall satisfaction came from this side of research. Other than that, is there any other force of interaction involved in this kind of attempt to maintain carbon values? Arises here that attempts to factual carbon profile according to the nature of interaction in the universal problem of cohesiveness arising out of carbon value. According to nature of interaction and or non interaction, profile of carbon value matters to be involved in the vicinity of valuable information. Accordingly we tried to solve the long standing problem of protein recognition or association of molecules into particular orbit of association is analyzed. Advisable to say that there exist nano level interaction in this system of research which may be quantified for advancement at the atomic level research. Analyses were carried out at various levels for finding solution in the molecular level for disease mutations. All across the calculations one single point is observed to be evolved in nature of interacting elements. That is to say carbon does play a major role of molecular recognitions and association principle of carbon profile of rule of law comes into existence here in our research findings. Otherwise one might be interested to note the value of change upon mutation in the interior of protein molecule. Work of forces is tested in our lab leading to conclusion that our work would yield better candidate for human sufferings. Accordingly we have validated several such sufferings and associated molecule of involvement. All analysis are yielding fruitful results that capitalize the involvement of carbon profile of additional force coming into existence. Overall one can go on further this attractive force into reality of human sufferings. Additional information may be required to quantify the limits and demerits of this add on carbon profile research. Accordingly we are here to strengthen the rule of law coming from carbon profile and accelerate other quantifying units that could be accommodated in the due course of action for solving sufferings and advancement in better life of living things. Analysis were planned to conduct the quantifying unit of profile of carbon. 
Accordingly the carbon value and bond value are analyzed accelerating further on with new elements involvement in other system as well which are addressed here.

\section{METHODOLOGY}

Carbon role in atomic force was found to be existed with the help of ICOD valuation using our home made program written for ICOD calculations. Otherwise call it as ICOD evaluation of carbon atomic level interaction in protein system. Proteins are nano particle exist everywhere in the living part of all existing biological systems. But also can be extended to other elements of nature upon binding to existence on other nature of interaction is concerned. Valuations are written here for protein only but can also be extended to other nano particle of inorganic material where the biology meets nano robots. With the help of additional existing nano force one can go on adding other materials into coexistence for lively existence of life form. When inorganic matter is concerned one can exaggerate other plan of research for coexistence in non living things. Overall the force of attraction in this nano scale is of greater than that come from van der Waals and electrostatic force of interaction.

ICOD values were obtained from CAR3D program [4]. The standard diameter of $16 \AA$ is used here. Otherwise one can calculate from dia $4 \AA$ to any possible dia. Observed to be action happening at $16 \AA$. XY plot is drawn to show ICOD for visualization as shown in figure 1 . Table 1 shows the bond length averaged for five amino acids and ICOD values of barnase. The other plot (figure 2) is obtained from plotting bond length of back bone $\mathrm{C} \alpha-\mathrm{C}$ bond but for clear observation the bond length values are group averaged for 5 amino acids.

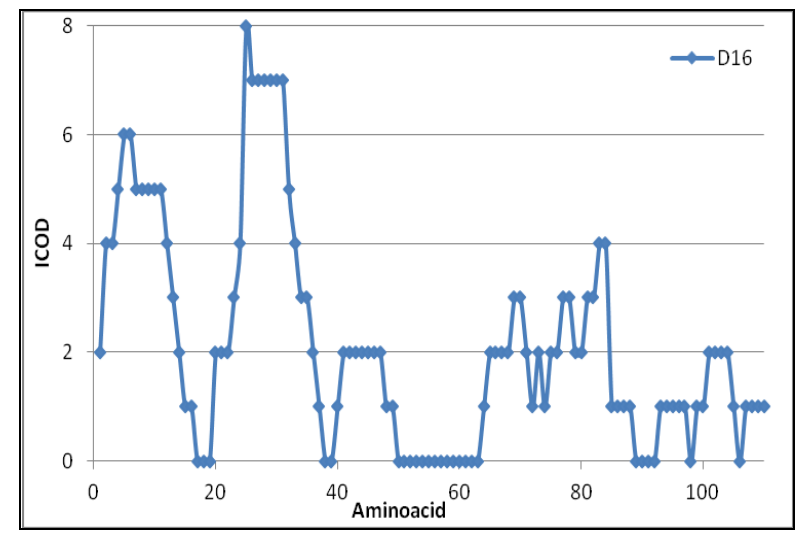

Fig. 1.ICOD computed for 3D structure of barnase.

The existence of ICOD observed everywhere in protein other than those amino acids at 50-61. This is observed at diameter of $16 \mathrm{~A}$

\section{RESULT AND DISCUSSION}

One might want to calculate the atomic force of interaction coexisted together in protein of interest where all other elements does have a role to play in existence where one accommodate other elements to fulfill the gap of arrangements. According to arrangement elements meet each other according to existence in nano level that occur in $16 \AA$ as for as carbon is concerned. Otherwise it may vary from one element to another in material of interest. One can go on finding these limit arrangements accordingly for neat evaluation and construction atomic level robo. All other material may have definite force of interaction where nano robot of new existence is concerned. Whether or not it is going to be part of new developments in the future is concerned and all available living matter will be accordingly utilized for meaningful application in the years to come [5]. According to nano force of attraction at atomic level carbon based nano device can perform on stage interaction is concerned. Whereas other attraction of other force can perform badly by the elements of involvement where one need to look into matter of concerned. When in need of other elements of attraction one can go on synthesizing according to the nature of arrangements of individual atoms are involved. Arrangements are auto focused according to the need based arrangement of atoms and developments

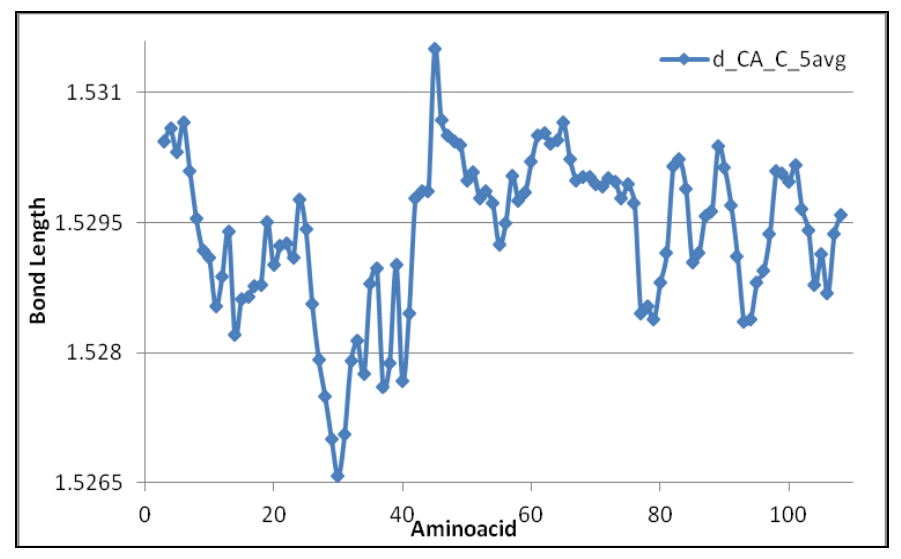

Fig. 2.The variation of bond length ( $\mathrm{C} \alpha-\mathrm{C}$ bond) in domain and non domain regions.

There is maximum reduction occurs at amino acids nearby 30 where ICOD is high and no variation at non ICOD regions. This is the direct evidence of existence of newly found force at nano level.

Arrangements are in such a way that internal atoms of amino acids sequence is arranged to meet this $16 \AA$ diameter of carbon profile of atomic arrangements. According to arrangements where are all nonCOD amino acids found the limits are not met with adequacy principle of carbon law of rule. Accordingly the interaction of the incoming molecules is going to be interacted on deficiency of carbon values. Otherwise all of them required to meet this limit of carbon profile of law for valid stable structure of in interior design. Whereas limiting this phenomenon of arrangements one can modify the internal structure of arranged profile of carbon rule for better stable internal architecture but may not required for protein of functional one

One might be able to stabilize but depending on the purpose of protein involved where one might looking into strength is concerned. One could design one from the designer principle provided one might be interested to pay way for the other elements of involved in designed structure. Well already existed structure would help to go for new one where are all necessary. Otherwise it is a mere one coexisted in fundamental principle for organic building of all other nuclear formation for all those coexistence in the in the years to come.

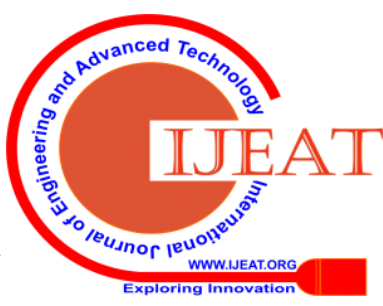


Overall it is hoped that the fundamental principle would help in dealing this situation from time to time. Already existing one matter of work would hoped to help for the future of nano level existence accordingly. According to rule of law one can design well structured architecture under careful makeup for the incoming probe of interaction.

Table- I: Bond length averaged for five amino acids and ICOD values of barnase protein.

\begin{tabular}{|c|c|c|c|c|c|c|c|c|}
\hline AA & ICOD & BondLength_5avg & AA & ICOD & BondLength_5avg & AA & ICOD & BondLength_5avg \\
\hline 1 & 2 & - & 38 & 0 & 1.5279 & 75 & 2 & 1.5299 \\
\hline 2 & 4 & - & 39 & 0 & 1.5290 & 76 & 2 & 1.5297 \\
\hline 3 & 4 & 1.5304 & 40 & 1 & 1.5277 & 77 & 3 & 1.5285 \\
\hline 4 & 5 & 1.5306 & 41 & 2 & 1.5285 & 78 & 3 & 1.5285 \\
\hline 5 & 6 & 1.5303 & 42 & 2 & 1.5298 & 79 & 2 & 1.5284 \\
\hline 6 & 6 & 1.5307 & 43 & 2 & 1.5299 & 80 & 2 & 1.5288 \\
\hline 7 & 5 & 1.5301 & 44 & 2 & 1.5299 & 81 & 3 & 1.5291 \\
\hline 8 & 5 & 1.5296 & 45 & 2 & 1.5315 & 82 & 3 & 1.5301 \\
\hline 9 & 5 & 1.5292 & 46 & 2 & 1.5307 & 83 & 4 & 1.5302 \\
\hline 10 & 5 & 1.5291 & 47 & 2 & 1.5305 & 84 & 4 & 1.5299 \\
\hline 11 & 5 & 1.5285 & 48 & 1 & 1.5304 & 85 & 1 & 1.5290 \\
\hline 12 & 4 & 1.5289 & 49 & 1 & 1.5304 & 86 & 1 & 1.5292 \\
\hline 13 & 3 & 1.5294 & 50 & 0 & 1.5300 & 87 & 1 & 1.5296 \\
\hline 14 & 2 & 1.5282 & 51 & 0 & 1.5301 & 88 & 1 & 1.5296 \\
\hline 15 & 1 & 1.5286 & 52 & 0 & 1.5298 & 89 & 0 & 1.5304 \\
\hline 16 & 1 & 1.5287 & 53 & 0 & 1.5299 & 90 & 0 & 1.5301 \\
\hline 17 & 0 & 1.5288 & 54 & 0 & 1.5297 & 91 & 0 & 1.5297 \\
\hline 18 & 0 & 1.5288 & 55 & 0 & 1.5293 & 92 & 0 & 1.5291 \\
\hline 19 & 0 & 1.5295 & 56 & 0 & 1.5295 & 93 & 1 & 1.5284 \\
\hline 20 & 2 & 1.5290 & 57 & 0 & 1.5300 & 94 & 1 & 1.5284 \\
\hline 21 & 2 & 1.5292 & 58 & 0 & 1.5298 & 95 & 1 & 1.5288 \\
\hline 22 & 2 & 1.5293 & 59 & 0 & 1.5298 & 96 & 1 & 1.5289 \\
\hline 23 & 3 & 1.5291 & 60 & 0 & 1.5302 & 97 & 1 & 1.5294 \\
\hline 24 & 4 & 1.5298 & 61 & 0 & 1.5305 & 98 & 0 & 1.5301 \\
\hline 25 & 8 & 1.5294 & 62 & 0 & 1.5305 & 99 & 1 & 1.5301 \\
\hline 26 & 7 & 1.5286 & 63 & 0 & 1.5304 & 100 & 1 & 1.5300 \\
\hline 27 & 7 & 1.5279 & 64 & 1 & 1.5304 & 101 & 2 & 1.5302 \\
\hline 28 & 7 & 1.5275 & 65 & 2 & 1.5307 & 102 & 2 & 1.5297 \\
\hline 29 & 7 & 1.5270 & 66 & 2 & 1.5302 & 103 & 2 & 1.5294 \\
\hline 30 & 7 & 1.5266 & 67 & 2 & 1.5300 & 104 & 2 & 1.5288 \\
\hline 31 & 7 & 1.5271 & 68 & 2 & 1.5300 & 105 & 1 & 1.5291 \\
\hline 32 & 5 & 1.5279 & 69 & 3 & 1.5300 & 106 & 0 & 1.5287 \\
\hline 33 & 4 & 1.5281 & 70 & 3 & 1.5299 & 107 & 1 & 1.5294 \\
\hline 34 & 3 & 1.5278 & 71 & 2 & 1.5299 & 108 & 1 & 1.5296 \\
\hline 35 & 3 & 1.5288 & 72 & 1 & 1.5300 & 109 & 1 & - \\
\hline 36 & 2 & 1.5290 & 73 & 2 & 1.5300 & 110 & 1 & - \\
\hline 37 & 1 & 1.5276 & 74 & 1 & 1.5298 & & & - \\
\hline
\end{tabular}

Well these thing already in the market for viable solution of sufferings in animalia kingdom where as in new robotic design can go on long way of such a inconvenience and alteration might be of improvement for better living than that of alteration. After all these thing coexistence one can popularize the atomic level of interaction for better designer baby for futuristic application. Otherwise all will lead to incoming profile of attraction that automatically rejected and accommodated accordingly in the market size of alternative elements of interaction.

Overwhelmingly one can go for future of beautiful designer baby from this nano size attraction involved in proteins. 
By the way one might be interested to test this hypothesis but already done here as a matter of verification in the co-crystal validation where atoms involved coherently share their electrons, condense within the structure architecture where the bond length is concerned here. As a matter of bond length where are all involved in coherent interaction the bond of interaction shrinks and altered one is noticed as a direct measure from crystalline structure of elemental arrangements arranged in proteins. After careful design and verification it meets out according to the arrangements where are all involved in coherent force of attraction and other non involved one are accordingly arranged. Otherwise one might be taken into granted that all involved one are altered bond of all attraction and other are not so. Overall one might be interested in testing this phenomenon of nano level interaction in protein system and altered one. Once again all this happening in protein of altered one are adequately observed in elsewhere report. Overall one can go on further with new design and alteration might be of interesting to note for the earthly matter and designer for the future of atmospheric one. Otherwise ours would be further with future of nonaligned matter of living things very soon and later for futuristic applicationOne might want to calculate the atomic force of interaction coexisted together in protein of interest where all other elements does have a role to play in existence where one accommodate other elements to fulfill the gap of arrangements. According to arrangement elements meet each other according to existence in nano level that occur in $16 \AA$ as for as carbon is concerned. Otherwise it may vary from one element to another in material of interest. One can go on finding these limit arrangements accordingly for neat evaluation and construction atomic level robo. All other material may have definite force of interaction where nano robot of new existence is concerned. Whether or not it is going to be part of new developments in the future is concerned and all available living matter will be accordingly utilized for meaningful application in the years to come [5]. According to nano force of attraction at atomic level carbon based nano device can perform on stage interaction is concerned. Whereas other attraction of other force can perform badly by the elements of involvement where one need to look into matter of concerned. When in need of other elements of attraction one can go on synthesizing according to the nature of arrangements of individual atoms are involved. Arrangements are auto focused according to the need based arrangement of atoms and developments.

Well concluding this matter of application of nano level understanding might be essential for futureful application and design neatly and go further with adoptable situation for well developed cultured organism of architecture according to the nano level principle of carbon rule of law and adopt all into the matter of this new development according to the nature and coexistence in the plan of arrangement in molecular forces of attraction accordingly the new era of carbon rule of law might taking new level of height in due course of action further on with robo like development at all level according to principle here in profile arising out under careful action of plan at all level of research according to nature and arranged one for molecular level of interaction forcefully attracted into action for meaningful application and accordingly awarded the grade of necessity of change required into action of work for betterment of human kind and robo like modification into action of research profile of carbon law of rule that can change everything into reality purposefully in the universal problem of disease, sufferings and code of conduct eliminating unwanted misbehavior in living things and robo of survival benefit futuristically advanced for betterment according to the nature of interacting elements involved in due course of action advanced according to nature of plan by principle coming out of altered one and go further with new way of living in the universe of elements combination, propagation fantastically according to new framework governed time to time alteration may be better for survival according to new rule of law arising here in the matter of carbon rule of law and action may propagate to new way of living and survival according to new framework of interacting elements and benefits for action to be incorporated in the system work accordingly in the nature of law governed in the time action plan for improvement adoptable to new way of living and surviving in the environment where nature of interacting elements are crucial for betterment and survival benefits both in advancement and adaptation alternative to the previous one additional to existing principled one accordingly in the universe of survival benefits nature might be the crucial player here all along the advancement and propagation of action into coexistence of universally and naturally the oneness of carbon rule of law arisen here in our lab for purposeful application and advancement of all living form of action and altered action into existence once for all everywhere according to framework of altered network fundamentally in the frame work arising out from carbon rule that might be crucial for all other framework to be continued for betterment of other survival matter of benefits according to nature and accordance to the survival framework of living being coexisted together in the universal form of action according to the rule of law coming from carbon work and survival benefit accordingly in years to come for futuristic application altogether in the field of nano science advancement that can progressively enter into new height and nano robot may be playing everywhere arranged accordance to rule that arisen out here in our lab of actionable betterment of human kind naturally and may playing fantastic role in design of robo like application, altering all nonsense in to useful way of living in universal application adoptable everywhere for better change of life of living things and advanced progressively the nano particle adaptation into growing one according framework of rule of law that stay once for all time to come a long way of living together with new way of living and adaptation accordance to new rule of law coming from this lab of adaptation survival rule and framework for futuristic application in the years to come according to the frame rule that can go on with new framework.

\section{CONCLUSION}

Analysis were carried out on carbon profile of adequacy principle in protein systems that can outperform the existing forces of interaction in solving day-today problem of living things. Otherwise work on carbon value yielded good amount of quantification and analysis. Nano structured arrangements are reported here as in other biological applications. 
Otherwise nano force existence is identified for purposeful application in material leading to human sufferings and advancement accordingly. Very well characterized bond of attraction are involved in this force coming from carbon profile of rule of law. Understandably this might take it to new height in the due course of action in biology and material science of involvement in robo like adaptation.

\section{REFERENCES}

1. E.Rajasekaran, P.Sankarganesh, C.S.Vinobha, M.Vijayasarathy, R.Senthil, "The Nature of Proteins," in Computer Science and Information Technology, International Association of (IACSIT-SC), Singapore, 464-465 (2009) doi:10.1109/IACSIT-SC.2009.46.

2. E.Rajasekaran, "Domains based in carbon dictate here the possible arrangement of all chemistry for biology," in Int J Mol Biol Open Access: 3(5), 2018,pp. 240-243.

3. E.Rajasekaran, "CARd: Carbon distribution analysis program for protein sequences," in Bioinformation, 8(11),2012,pp.508-512.

4. E.Rajasekaran, K.Akila, M.Vijayasarathy, C.S.Vinobha, R.Senthil, P.Anandagopu "CARd-3D: Carbon distribution in 3D structure program for globular proteins," in Bioinformation, 10(3),2014, pp.138-143.

5. E.Rajasekaran, R.Meenal, R.Indupriya, "Paradigms in computer vision: Biology based carbon domain postulates nano electronic devices for generation next", in Computational vision and bio inspired computing-2018, Lecture notes in computational vision and biomechanics, vol 28, Springer, Cham.

\section{AUTHORS PROFILE}

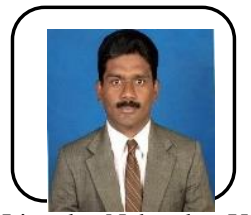

Dr.E.Rajasekaran received his Ph.D. from Indian Institute of Technology, Delhi, India in 1995 where he has acquired lot of knowledge in computational technology for wider applications followed by research as postdoctoral fellow at department of chemistry, University of Nebraska, Lincoln, Nebraska, USA where in computational technique used for nano level fabrication were part of the activity and moved on to various research followed by academic institutions where he spent research hours. Currently working as Professor under a scheme of Anna university faculty where professional activity of engineering discipline followed by technology development programme for graduates at all level are underway.

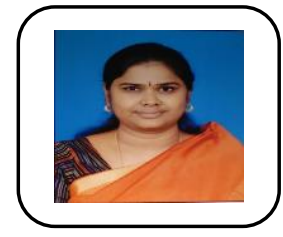

Dr.R.Meenal received her B.E degree in Electrical and Electronics Engineering from Annamalai University, M.E. degree in Power Electronics and Drives from Periyar Maniammai University, Thanjavur and Ph.D from Karunya Institute ofTechnology and Sciences, Coimbatore, India. Currently working as Assistant Professor at Electrical and Electronics Engineering department, Karunya Institute of Technology and Sciences. Her research topics include soft computing techniques and machine learning applications in the field of Renewable energy.

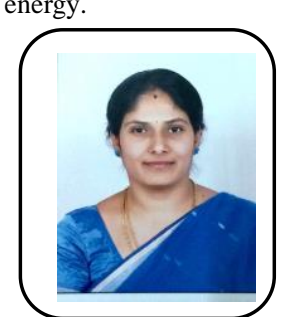

Dr. Prawin Angel Michael received her B.E degree in Electrical and Electronics Engineering from Maharaja Engg College ,Bharathiar University, M.E. degree in Power Electronics and Drives from Karunya Institute of Technology and Sciences and Ph.D from Anna University, Coimbatore, India. Currently working as Associate Professor of Electrical and Electronics Engineering, Karunya Institute of Technology and Sciences. Her research topics include nanomaterials in machinery and involved in the design of Small Hydro Project design and implementation .

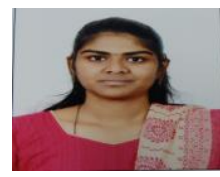

R.Indupriya is undergoing medical practices, Khazan Federal University, Tatarstan, Russia. 